
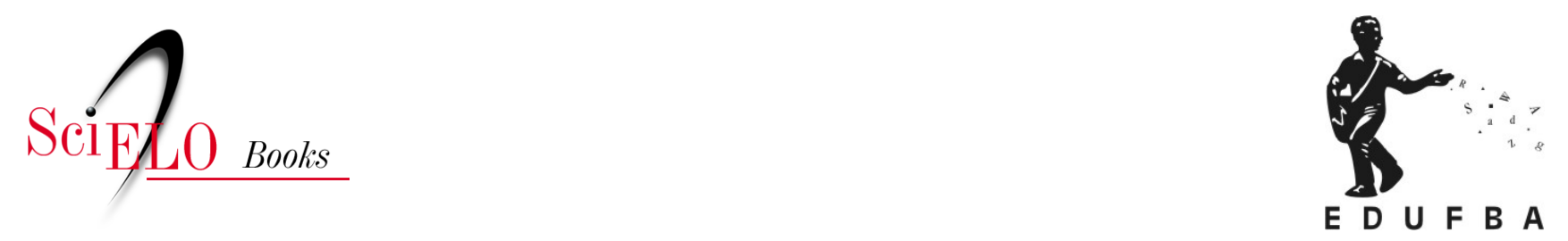

\title{
A infância como objeto de análise política em saúde
}

\author{
Monique Azevedo Esperidião \\ Carla Soares \\ Carolina Rodrigues \\ Marta Caires de Souza \\ Raquel Malheiros \\ Andréa Montalvão \\ Laiana Behy
}

ESPERIDIÃO, M.A., SOARES, C., RODRIGUES, C., SOUZA, M.C., MALHEIROS, R., MONTALVÃO, A., and BEHY, L. A infância como objeto de análise política em saúde. In: TEIXEIRA, C.F., comp. Observatório de análise política em saúde: abordagens, objetos e investigações [online]. Salvador: EDUFBA, 2016, pp. 305-338. ISBN 978-85-232-2021-1. https://doi.org/10.7476/9788523220211.0010. \section{International license.}

All the contents of this work, except where otherwise noted, is licensed under a Creative Commons Attribution 4.0

Todo o conteúdo deste trabalho, exceto quando houver ressalva, é publicado sob a licença Creative Commons Atribição $\underline{4.0}$. 
Monique Azevedo Esperidião, Carla Soares, Carolina Rodrigues, Marta Caires de Souza, Raquel Malheiros, Andréa Montalvão e Laiana Behy

\section{A INFÂNCIA COMO OBJETO DE ANÁLISE POLÍTICA EM SAÚDE}

\section{Introdução}

As políticas de atenção à saúde da criança, no Brasil, vêm sofrendo transformações em função de sua inserção histórica, dos avanços do conhecimento científico e da constituição de um espaço médico especializado, bem como de influências de organismos internacionais, configurando-se no interior de um espaço social que envolve o engajamento de distintos agentes sociais. A mortalidade infantil ainda vem sendo apontada como grande desafio para o país e a tomada deste tema pelo Estado brasileiro reveste-se de uma perspectiva desenvolvimentista, sendo a saúde das crianças a garantia da prosperidade da nação. (ARAÚJO et al., 2014)

Em geral, os estudos sobre as políticas de saúde voltadas para a infância analisam seu impacto na mortalidade infantil, a sua implementação bem como a qualidade do cuidado prestado. Alguns trabalhos mostram, contudo, que a sua formulação responde a determinações históricas e estruturais que têm sido pouco investigadas no nosso meio. (WARDE, 2007) O acompanhamento dessas políticas 
com o objetivo de produzir conhecimento capaz de contribuir para o seu aperfeiçoamento requer ,além de estudos avaliativos, a identificação desses processos sociais subjacentes.

Por essas razões, a construção e implementação de políticas voltadas para "proteção à infância" devem ser analisadas à luz das transformações nas relações entre o processo de especialização do campo médico e a questão da medicalização da infância (medicalização de doenças, incluindo transtornos do comportamento e escolares; e de práticas sociais com intervenções sobre a família, como a inculcação da higiene nas mães de famílias pobres).

Faz-se necessário analisar a constituição da infância como um problema das sociedades contemporâneas que requer intervenção estatal, tendo como resposta as políticas de luta contra a mortalidade infantil, a emergência da puericultura e de uma medicina da primeira infância e da atenção ao recém-nascido.

\section{Políticas de combate à mortalidade infantil}

A chamada "morte menina" (VAILATI, 2010), outrora esperada no Brasil dos oitocentos, há quase dois séculos, vem sendo temida e enfrentada pelo Estado e sociedade brasileiros. Seguramente, inúmeras conquistas são hoje festejadas - o país conseguiu exceder as Metas do Milênio da Organização das Nações Unidas (ONU), de redução de dois terços da mortalidade infantil de 1990 até 2015, em 2011, quatro anos antes do prazo assumido perante a organização. Além da redução na mortalidade infantil, o país vem apresentando resultados satisfatórios na queda do déficit de altura de crianças menores de cinco anos de idade e na melhoria ao acesso aos serviços de saúde, com coberturas quase universais para imunização e atendimento hospitalar ao parto. (VICTORA et al., 2011) Contudo, o Brasil enfrenta ainda os reflexos da perversa iniquidade regional e socioeconômica que marca o país e, em bolsões de miséria, perdem-se crianças antes de completarem o primeiro ano de vida. (VICTORA et al., 2011) 
Até o início do século XX, a atenção dispensada às crianças tinha um caráter filantrópico, assentada em propostas da puericultura. Surgiram as primeiras instituições de assistência e proteção à infância, bem como institutos profissionalizantes voltados às crianças doentes e abandonadas. (NOVAES, 1979)

As primeiras políticas organizadas pelo Estado brasileiro dirigidas à saúde das crianças remontam à criação do Estado Novo, pelo presidente Vargas. Em 1940, foi instituído o Departamento de Saúde da Criança, como forma de proteção social e melhoria das condições de vida e saúde da nascente classe trabalhadora no país. Tratava-se da organização de serviços, como creches, junto às fábricas. Tal departamento ocupou-se por quase 30 anos de uma série de ações programáticas com baixa integração. (TYRREL, 1997)

Ainda na década de 1970, sob a influência dos movimentos da medicina preventiva e comunitária, a importância da saúde materno -infantil foi reafirmada, mas as políticas implantadas tiveram pequeno alcance uma vez que o modelo assistencial vigente conformava-se pela divisão entre a saúde pública e a assistência médica individual com forte exclusão social. Em 1973, foi lançado o Programa Nacional de Imunizações (PNI), com o objetivo de coordenar as ações de imunizações, até então de caráter episódico e de reduzida cobertura. Tratava-se de experiências de caráter vertical, centralizadas e com viés tecnocrata. (TYRREL, 1997) O Programa de Atenção à Criança, implantado em 1976, resultou em um atendimento público racionalizador e burocrático. (ZANOLLI; MERHY, 2001)

Na década de 1980, já sob influência das lutas trazidas pelo movimento da Reforma Sanitária Brasileira, surge o Programa Nacional de Incentivo ao Aleitamento Materno, de 1981. Considerado exitoso, tinha a finalidade de incentivar o aleitamento materno e reduzir o desmame precoce. (BRASIL, 1991)

Em 1984, foi implantado o Programa de Assistência Integral à Saúde da Criança (PAISC), organizado como estratégia de combate à mortalidade infantil, considerada elevada, com ações voltadas para o enfrentamento às adversidades nas condições de saúde das 
crianças. (BRASIL, 1995) O PAISC representou assim uma iniciativa para promover a saúde de forma integral, priorizando agravos e crianças mais vulneráveis e propondo a expansão da cobertura. As ações estavam centradas no acompanhamento do crescimento e desenvolvimento, incentivo ao aleitamento materno, controle das doenças diarreicas, controle das infecções respiratórias agudas e na imunização. Surge, nesse período, o primeiro Cartão de Saúde da Criança. A abordagem epidemiológica desse programa encontravase relacionada à explicação do binômio desnutrição-infecção na estrutura de morbimortalidade infantil, sendo evidenciada uma cadeia de determinantes sociais ligados ao quadro de precárias condições de vida a que estava submetida a maioria das crianças brasileiras. (FIGUEIREDO; MELLO, 2007)

Com a criação do Sistema Único de Saúde (SUS), nos anos 1990, a saúde passa a ser assegurada como direito dos brasileiros e surge uma nova concepção organizativa do sistema de saúde, numa compreensão descentralizada, hierarquizada e regionalizada. A saúde da criança ganha novas perspectivas e impulsos. A consolidação do Programa Agentes Comunitários de Saúde (PACS) e do Programa Saúde da Família (PSF), especialmente nos municípios de pequeno porte das regiões Norte e Nordeste, foi decisiva para ampliação do acesso aos serviços com repercussões diretas à saúde das crianças. Posteriormente, com expansão da Estratégia Saúde da Família (ESF), essa conexão ficará mais forte. (FIGUEIREDO; MELLO, 2007)

Em 1995, o Ministério da Saúde (MS) lançou o Projeto de Redução da Mortalidade Infantil (PRMI) (BRASIL, 1995) com a finalidade de alcançar as Metas da Cúpula Mundial em Favor da Infância. O projeto visava a redução dos óbitos infantis e a melhoria da situação de saúde das crianças por meio da articulação intersetorial entre distintos programas governamentais, articulação com organizações nãogovernamentais, sociedades científicas, conselhos de secretários de saúde e a sociedade, além de cooperação com organismos internacionais, o Fundo das Nações Unidas para a Infância (Unicef) e a Organização Pan-Americana da Saúde (Opas). 
Em 1997, inicia-se, primeiramente em quatro estados (Pará, Pernambuco, Sergipe e Ceará), a iniciativa Atenção Integrada às Doenças Prevalentes na Infância (AIDPI), promovida pela OMS, Opas e Unicef. A AIDPI tinha por objetivo a redução da mortalidade em crianças menores de cinco anos e a organização da assistência à criança na atenção primária à saúde, enfatizando para além das ações assistenciais, propostas voltadas para promoção da saúde, prevenção de doenças e agravos. Em 2001, todos os estados e o Distrito Federal já haviam implantado a AIDPI. (BRASIL, 2002a)

Ainda no âmbito da atenção básica à saúde, foi lançado pelo MS, em 2002, o Caderno de atenção básica - Saúde da Criança: acompanhamento do crescimento e desenvolvimento infantil (BRASIL, 2002a), que reunia medidas para o crescimento e o desenvolvimento saudáveis. Tais medidas deveriam ser integradas ao AIDPI.

No ano de 2004, o MS reconheceu aravidade das taxas de mortalidade materna e neonatal brasileiras, entendendo que estas mortes representam formas de violação aos direitos humanos de mulheres e crianças. Por essa razão, propôs o Pacto pela Redução da Mortalidade Materna e Neonatal, cujo objetivo era criar soluções sustentáveis e dividir responsabilidades entre entes federativos e sociedade civil contra a mortalidade materna e neonatal. (BRASIl, 2004a)

Ainda em 2004, o MS apresentou a Agenda de Compromissos com a Saúde Integral da Criança e a Redução da Mortalidade Infantil, que visava à criação de uma rede única integrada de assistência à criança. $\mathrm{O}$ documento propunha uma série de ações para superar a falta de articulação entre os diversos níveis de atenção à saúde, garantindo a continuidade e a efetividade dos cuidados prestados às crianças. Também em 2005 foram criados os comitês locais para prevenção da mortalidade infantil. (BRASIL, 2004b)

Em 2006, a redução da mortalidade infantil foi ratificada como política de governo e incluída no Pacto pela Vida, expressando compromissos sanitários com a saúde da criança. (BRASIL, 2006) Em 2008, no Programa Mais Saúde, eixo "Promoção da Saúde", destacase a política "Brasileirinhos Saudáveis: primeiros passos para o de- 
senvolvimento nacional", que previa um conjunto de ações voltadas para a promoção da saúde e o acompanhamento de gestantes e crianças de zero a cinco anos. (BRASIL, 2008)

Em 2012, foi lançado o Programa Brasil Carinhoso que destacou o compromisso do Governo Federal em fortalecer estratégias para o desenvolvimento integral da primeira infância brasileira, envolvendo os ministérios da Saúde, Educação e Desenvolvimento Social e Combate à Fome. O Brasil Carinhoso previu a ampliação do Programa Bolsa Família para crianças até seis anos, maior acesso a creches por meio do Programa Saúde na Escola, maior prevenção de anemia por deficiência de ferro e prevenção da deficiência de vitamina A, com a suplementação oferecida nos centros de saúde como importante ação que impacta diretamente no crescimento da criança, na capacidade de aprendizagem e no desenvolvimento cognitivo. (BRASIL, 2015a)

Apenas em novembro de 2014, foi aprovada a Política Nacional de Atenção Integral à Saúde da Criança (PNAISC), bastante feste310 jada e comemorada pelos atores envolvidos na luta em favor da infância. A política possibilita a integração dos programas existentes e tem em vista a atenção integral às crianças, especificamente para primeira infância. A política foi proposta pela Coordenação de Saúde da Criança e Aleitamento Materno (CGSCAM) do MS, em parceria com a equipe da Estratégia Brasileirinhas e Brasileirinhos Saudáveis, do Instituto Nacional de Saúde da Mulher, da Criança e do Adolescente Fernandes Figueira(IFF).

A PNAISC procura implementar os direitos das crianças, previstos na Constituição Federal e no Estatuto da Criança e do Adolescente (ECA), contemplando crianças e adolescentes menores de 16 anos com especial atenção à primeira infância e às crianças em situação de maior vulnerabilidade, para reduzir a morbimortalidade e favorecer um ambiente facilitador à vida e ao pleno desenvolvimento.

No âmbito da sociedade civil, algumas iniciativas recentes merecem ser destacadas, como a Rede Nacional Primeira Infância (RNPI). A RNPI iniciou suas ações em 2007 e hoje compreende 
mais de 160 organizações de todas as regiões do Brasil mobilizadas pela causa da infância. Ela foi responsável pela elaboração do Plano Nacional pela Primeira Infância, priorizando todos os direitos da Primeira Infância, com metas até 2022. O plano foi aprovado pelo Conselho Nacional pelos Direitos da Criança (Conanda), em 2010, e acolhido pela Secretaria de Direitos Humanos da Presidência. Em 2014, foi criado também o Comitê de Especialistas e Mobilização Social para o Desenvolvimento na Primeira Infância, no MS.

Finalmente, cabe refletir que as políticas que tomam a infância como objeto são majoritariamente setoriais, limitadas a intervenções pontuais e fragmentadas. O lançamento da política nacional, apenas em dezembro de 2014, ilustra como as ações encontravam-se pulverizadas e desconexas. Investimentos estruturais, com ação coordenada sobre as condições de vida, em especial na educação e na geração de emprego para as famílias, representam perspectivas mais promissoras e com maior impacto. (VICTORA, 2001) De fato, trabalhos recentes mostram efeito positivo de programas sociais como Bolsa Família (RASELLA etal., 2013) e da ESF na redução da mortalidade infantil. (AQUINO; OLIVEIRA; BARRETO, 2009) Ao lado do desafio de redução das desigualdades, persiste a necessidade de redução do elevado número de cesarianas, nascimentos pré-termo e abortos inseguros. (VICTORA et al., 2011)

\section{Reflexões acerca do conceito de infância}

A conceitualização da infância tem sido objeto de luta entre diferentes campos científicos, teorias e instituições. Na antiguidade, a infância não existia enquanto objeto de discurso ou de preocupações específicas, sendo a criança considerada apenas um adulto em miniatura. Conforme destaca Ariès (1986), não havia um sentimento de infância. A preocupação e a conceitualização da infância surge com a modernidade, porém não tinha o cuidado à criança como um fim em si mesmo, mas com o adulto do futuro. A criança era, portanto, conceitualizada pelo seu devir. 
Nos séculos XIX e XX, surge a infância científica, com o reconhecimento desta como uma etapa do desenvolvimento e objeto de estudo de diversas áreas do saber. Surgem então múltiplas prescrições para cuidados nesse período: normas de higiene, campanhas de amamentação, instituições de atendimento, entre outros. (BARBOSA, 2006)

O conceito biológico, predominante no senso comum e em políticas públicas, demarca a infância como etapa do desenvolvimento marcada por um intervalo delimitado cronologicamente ( 0 aos 12 anos, 0 aos 9 anos) ou por maturação anatômica e fisiológica (nascimento à entrada na puberdade). Essa perspectiva é a mais utilizada por documentos institucionais e políticas públicas, pelo menos no que tange à definição. Nesse sentido, o documento nacional mais recente (BRASIL, 2015a) se refere à criança como a pessoa na faixa etária de zero a nove anos. A definição do PNAISC difere de documentos nacionais e internacionais anteriores, uma vez que o ECA (BRASIL, 1990) define o limite etário do fim da infância aos 12 anos e a Convenção dos Direitos da Criança (ONU, 1989) o coloca aos 18 anos.

Conceitos frequentemente utilizados pela psicologia do desenvolvimento e pela educação modernas partiram também da perspectiva de infância como fase do desenvolvimento, na qual a criança, progressivamente e dadas as condições necessárias, adquire as características de um adulto. Assim, a criança foi teorizada da perspectiva do seu desenvolvimento cognitivo, moral, emocional, entre outros.

Buscando um conceito de infância para a pós-modernidade, a educação, a psicologia e áreas do conhecimento, fortemente encabeçadas pela sociologia da infância propõem rompimento com o paradigma de fragilidade, inocência, dependência e incapacidade que costumavam nortear essa conceitualização. Passam a reconhecer a criança como sujeito ativo, coconstrutor de conhecimento, identidade e cultura. (BARBOSA, 2006; JAVEU, 2005; QVORTRUP, 2010; SARMENTO, 2005) 
Nesse sentido, iniciam por problematizar "a negatividade constituinte da infância” (SARMENTO, 2005, p. 368), que tende a definir essa categoria pelas suas faltas: infância, idade do não falante ou inarticulado; juridicamente inimputável e socialmente interditado. Embora essas características e interdições sejam a base da proteção da criança e, portanto, avanços civilizatórios, o autor as utiliza para enfatizar o fato de que os esforços de definição e descrição da condição infantil partiram do que lhes falta, em lugar de buscar o que positivamente lhes define. Destaca o autor que há “ [...] um efeito simbólico de conceptualização e representação sócio-jurídica da infância pela determinação dos factores de exclusão e não, prioritariamente, pelas características distintivas ou por efectivos direitos participativos”. (SARMENTO, 2005, p. 368)

Outro ponto importante da conceitualização pós-moderna de infância é a busca desses teóricos por uma diferenciação conceitual entre infância e criança. Considera-se infância, portanto, como uma categoria social do tipo geracional, e criança como sujeito concreto integrante dessa categoria, e também ator social com outro pertencimentos, como classe e gênero. (QVORTRUP, 2010; SARMENTO, 2005)

Numa diferenciação similar, Javeu (2005), vai mais além, com as categorias criança, infância, crianças, às quais atribui a diferentes áreas do conhecimento. $\mathrm{O}$ autor atribui o termo "criança" ao campo da psicologia, cujas disciplinas relacionadas, de acordo com o autor, legitimaram um discurso sobre etapas de desenvolvimento da criança. A "criança”, nesse caso, é pensada como objeto abstrato “[...] destinado a passar por níveis diversos e sucessivos na aquisição de competências, cada um deles constituindo uma etapa na fabricação da personalidade dos indivíduos". (JAVEU, 2005, p. 382)

O termo "infância" é atribuída pelo autor à perspectiva demográfica, na qual é colocada lado a lado com outras faixas etárias (adolescência, idade adulta, terceira idade), e caracterizada pela sua improdutividade. O termo "crianças" pertence ao último campo semântico listado pelo autor, o da antropologia, que tem como foco o 
grupo de indivíduos no seu território, com suas estruturas e modelos de comportamento específicos. (JAVEU, 2005)

Essas perspectivas emergentes sobre criança e infância trazem consigo mudanças nas reflexões sobre aspectos heterogêneos e homogêneos desta geração. Compreende-se a infância como historicamente construída, através de um longo e continuamente atualizado processo que elabora as bases ideológicas e normativas do lugar da criança na sociedade. Participam desse processo variações demográficas, relações econômicas, políticas públicas, dispositivos simbólicos e estilos de vida. (QVORTRUP, 2010;SARMENTO, 2005) Nesse sentido, discute-se que se trata de "infâncias", no plural, pela forma como a categoria geracional em questão está imbrincada com outras de ordem étnica, social, econômica e de gênero.

\section{Estado da arte sobre políticas de saúde da criança}

A criança é um ser que vive intensas transformações, tanto biológicas quanto afetiva, psicológicas e sociais, desde sua vida intrauterina até o seu completo desenvolvimento que culmina com o final da adolescência. Portanto, pensar na saúde de seres que vivem uma série de transformações tão complexas é adquirir uma visão integral e multidisciplinar da saúde. No entanto, a criação e implementação de políticas públicas voltadas para essa faixa etária enfrenta uma série de dificuldades, inclusive porque a compreensão da infância foi bastante controversa ao longo dos séculos.

Para Araújo e colaboradores (2014), a criança ocupou diferentes papéis na sociedade, que, ao longo dos anos, sofreu inúmeras transformações. Essas modificações conduziram ao enfrentamento de diversas situações que repercutiam em sua qualidade de vida e, consequentemente, na saúde. Desse modo, com o transcorrer do tempo, a criança adquiriu um caráter de sujeito social assumindo as características próprias de acordo com o seu período de vida.

Até o século XVI, a sociedade não percebia as particularidades da infância e as crianças eram tratadas da mesma forma que os adul- 
tos. Sendo assim, não havia uma compreensão do significado da infância para o desenvolvimento humano. A partir do século XVII, a criança passou a ocupar um espaço social, uma vez que as famílias passam a demonstrar sentimentos familiares pelos infantes. No entanto, a preocupação com a saúde e o bem estar da criança só é percebido pela sociedade a partir do século XX. (ARAÚJO et al., 2014)

Em 1920, as entidades públicas e privadas demonstraram preocupação com a saúde infantil uma vez que as crianças eram dependentes dos trabalhadores das fábricas, sobretudo das mulheres. Assim, nesse período, passou-se a discutir sobre o aleitamento materno, uma vez que a amamentação reduzia o adoecimento de crianças ainda na primeira infância, o que permitia que suas mães cumprissem a jornada de trabalho com mais regularidade. (ARAÚJO et al., 2014)

Entre os anos de 1930 e 1940, alguns programas voltados à maternidade e infância foram criados de acordo com as propostas do Departamento Nacional da Criança. No entanto, essas ações voltadas para a gravidez e a primeira infância possuíam um caráter individualizado, curativo e voltado para um modelo de saúde altamente hospitalocêntrico. Portanto, não considerava a importância de ações coletivas com foco na promoção da saúde e não apenas na prevenção, e tampouco que as características individuais repercutem no quadro de saúde e bem estar de toda uma sociedade e não apenas de uma pessoa ou família. (ARAÚJO et al., 2014; ROCHA et al., 1998; SOUZA; OLIVEIRA, 2004)

Para Souza e Oliveira (2004), partir de 1930, a política de saúde nacional foi centralizada em dois subsetores: a saúde pública e a medicina previdenciária, sendo a saúde pública mais prevalente. Com o desenvolvimento do ensino da pediatria, a assistência à criança foi institucionalizada através da puericultura e da assistência médica privada. Porém, a política social tinha como pilar central o assistencialismo de caráter populista.

Ramos e Maia (1999) afirmaram que o desenvolvimento de políticas de saúde é realizado através do planejamento social de políti- 
cas de saúde e, sendo assim, fazem parte de um ato político e social. Portanto, os planejadores de políticas públicas devem não apenas exercer o seu papel, mas também, adquirir uma competência política.

Crianças e adolescentes estão em pleno desenvolvimento e, a depender de cada fase de crescimento, possuem necessidades específicas. A condição de existência e a qualidade de vida de uma criança estão diretamente relacionadas às suas fragilidades e riscos de adoecimento. No entanto, as disparidades apresentadas nessa faixa etária transcendem as diferenças biológicas do organismo uma vez que as desigualdades e iniquidades sociais são determinantes igualmente relevantes para o amadurecimento pleno de uma criança. (ROCHA et al., 1998)

Dessa forma, o ECA, lei 8.069 de 1990, regulamenta que a criança possui direito à vida e à saúde desde o momento da concepção até a adolescência e que cabe ao Estado, por intermédio do SUS, promover programas de assistência médica e odontológica para prevenção das comorbidades recorrentes na população infantil e campanhas 316 de educação sanitária nas escolas e para os familiares. (CABRAL; AGUIAR, 2003)

No entanto, analisando as políticas de saúde voltadas para a infância, percebemos através de investigações mais atuais que a sociedade ainda atribui, de forma centralizada, os cuidados da saúde em dois polos: a rede hospitalar e os serviços nas comunidades. (ROCHA et al., 1998)

Oliva e Kauchakje (2009) realizaram uma pesquisa sobre os direitos de crianças e adolescentes com ênfase na efetivação de políticas voltadas para a educação, saúde e assistência social nas capitais brasileiras e perceberam que há uma heterogeneidade de ações entre as capitais, entretanto, políticas públicas com ações voltadas para a educação ainda são maioria quando comparadas com as demais políticas públicas do estudo. Ainda segundo essa pesquisa, os objetivos voltados à política de saúde são os menos mencionados, e quando surgem, aparecem em maior quantidade no sul do país. 
Por meio desse estudo, podemos perceber que a efetivação de políticas públicas voltadas para a saúde da criança e do adolescente ainda são negligenciadas perante o governo e a sociedade, apesar dos esforços e dos debates constantes sobre essa temática no nosso país.

Ao avaliar o uso de serviços da atenção primária à saúde pela população infantil do estado do Maranhão, Cunha e colaboradores (2013) observaram que praticamente todas as variáveis do estudo obtiveram um incremento positivo em relação ao estudo da década passada. O acesso ao serviço público ascendeu cerca de $17 \%$ e as consultas de puericultura no primeiro ano de vida e antes do primeiro mês de vida cresceram em média $10 \%$. Assim, analisando as políticas de saúde voltadas para a infância, há uma década, observou-se a melhoria em alguns indicadores de atenção à saúde da criança do estado do Maranhão, que possivelmente está relacionada aos processos de implementação e aperfeiçoamento da ESF.

Segundo uma pesquisa realizada por Finkler e colaboradores (2014), a maioria dos atendimentos realizados às crianças na atenção básica ocorre por meio de consultas médicas onde o foco principal são as situações de agudização dos processos de doença. Assim, a saúde da criança não contempla a integralidade do cuidado e propõe um acesso à atenção primária a saúde fragilizado, fragmentado e descoordenado.

De acordo com Pimenta, Rocha e Marcondes (2015), as políticas públicas brasileiras voltadas para intervenções na obesidade infantil conduzem suas ações na prevenção da doença e na promoção de uma vida futura mais saudável através de ações de educação, orientação e estímulo de atividades físicas e de uma dieta equilibrada. Por conseguinte, os autores defendem que as políticas públicas voltadas para intervenções na obesidade infantil não devem se restringir apenas ao âmbito da saúde. Essas ações, portanto, devem possuir um caráter intersetorial abrangendo o máximo de setores do serviço público.

A Política Nacional de Alimentação e Nutrição (PNAN) e a Política nacional de Promoção à Saúde (PNPS) possuem ações es- 
pecíficas, segundo os determinantes sociais da saúde. A PNAN enfatiza a promoção de uma vida saudável bem como melhoria das condições de alimentação, nutrição e saúde da população brasileira, mediante práticas de alimentação saudáveis, vigilância alimentar e nutricional e prevenção e cuidado dos agravos relacionados à nutrição e a dieta. (PIMENTA; ROCHA; MARCONDES, 2015)

Já as ações da PNPS possuem um foco maior na capacidade de autorregulação do indivíduo com relação ao seu próprio cuidado em parceria com o Estado, que jamais deve se ausentar da sua responsabilidade. Assim, a PNPS promove a divulgação e implantação da política nacional de promoção à saúde, alimentação saudável e prática de exercícios físicos, prevenção e controle do tabagismo, que se relacionam diretamente com a obesidade. (PIMENTA; ROCHA; MARCONDES, 2015)

Além disso, o Programa de Saúde na Escola (PSE) e a Câmara Interministerial de Segurança Alimentar e Nutricional (Caisan), que lançou o Plano Intersetorial de Prevenção e Controle da Obesidade, também possuem estratégias para prevenir e controlar a obesidade infantil. Porém, Pimenta, Rocha e Marcondes (2015) pontuam ainda que a não eficácia de medidas corretivas, preventivas e de promoção no tocante à obesidade infantil são decorrentes também do sistema político e econômico vigente no país e que a compreensão da obesidade infantil no âmbito das Políticas Públicas - uma macro perspectiva - é de extrema relevância.

Para Couto, Duarte e Delgado (2008), que descreveram e analisaram a situação atual de desenvolvimento da política pública brasileira de saúde mental infantil e juvenil, os dados obtidos no presente estudo sugerem a existência de três grandes desafios a serem enfrentados pelo setor específico da saúde mental infantil e juvenil: inicialmente, através da situação de implantação de dispositivos para a atenção à saúde mental infantil e juvenil, em que se faz necessária a expansão dos diferentes serviços que compõem a rede de cuidados; o número insuficiente de Centros de Atenção Psicossocial (CAPSi), assim como o de ambulatórios e demais dispositivos de saúde men- 
tal, sobretudo aqueles que são voltados especificamente para a infância e adolescência.

Ainda segundo Couto, Duarte e Delgado (2008), essas necessidades estão presentes em todas as regiões do país, sobretudo no Norte e Centro-Oeste. No entanto, o enfrentamento desse problema depende da conscientização de gestores e da sociedade sobre a relevância da saúde mental para a política de saúde da infância.

A criação do protocolo dos Indicadores Clínicos de Risco para o Desenvolvimento Infantil (IRDI) foi uma grande contribuição da psicologia atual para as políticas de saúde, pois articula entre os âmbitos sociais e psíquicos na construção de uma política pública relacionada com o cuidado no desenvolvimento. Sendo assim, a aplicação do protocolo de IRDI e dos seus fundamentos tem sido uma excelente experiência para os profissionais da atenção básica que passam a compreender a singularidade dos indivíduos envolvidos na avaliação e nas orientações realizadas durante todo o processo. Por conseguinte, o IRDI representa um grande avanço para as ações de política de saúde da criança. (LERNER et al., 2013)

Apesar da existência de vários métodos legais de proteção à criança e ao adolescente, a violência, muitas vezes, não é percebida como tal, pois se traveste de forma disciplinatória ou punitiva e, portanto, não compreendida como uma forma de agressão. No entanto, a violência doméstica pode ser considerada uma questão de saúde pública, pois está relacionada tanto com o desenvolvimento social quanto econômico, além de ir contra os direitos humanos. Nos últimos anos, a violência adquiriu um caráter endêmico, e por esse motivo é preciso formular projetos que previnam esse problema social.

Apesar de ter sido implementada uma Política Nacional de Redução da Morbimortalidade por Acidentes e Violência, tornou-se obrigatório a notificação de todos os casos, suspeitos ou confirmados de violência com objetivo de iniciar um processo para interromper com as atitudes do agressor, além de promover cuidados sociais e sanitários voltados às vítimas. No entanto, muitos profissionais da área de saúde são despreparados e deixam de realizar as notificações 
por acreditarem que esse é um problema jurídico e não de saúde pública. Portanto, apesar de haver uma política para redução da violência contra criança, existem diversas lacunas nas notificações e identificações desses casos pelos profissionais e, por conseguinte, é preciso criar mais programas permanentes de educação nesse âmbito.

Para Marques (2010), ainda faltam muitas conquistas dentro das políticas públicas voltadas para a infância. Apesar de ter acontecido alguns avanços na criação de um sistema de garantia de direitos, as infraestruturas institucionais ainda são bastante precárias e o não cumprimento do que a Constituição e o ECA instituem há décadas gera grandes deficiências na efetivação do atendimento intersetorial e na integração do atendimento à criança e adolescentes.

Desse modo, ao realizarmos uma análise das políticas públicas voltadas para a saúde da criança instituída nesses últimos anos no nosso país, percebemos que, apesar dos esforços para melhorar a qualidade de vida das crianças, resultando consequentemente na diminuição das taxas de mortalidade e desnutrição infantil, ainda 320 presenciamos muitos contrastes dentro do Brasil. A região Nordeste, por exemplo, é detentora da maior taxa de mortalidade infantil do país, uma vez que concentra metade dos óbitos ocorridos nessa faixa etária no Brasil. (BRASIL, 2002b)

Assim, espera-se que, com a criação da Política Nacional de Atenção à Saúde da Criança, sejam fortalecidas ações integradas para atingir essa população alvo não apenas no combate a mortalidade e desnutrição infantil, mas auxiliando a sociedade a compreender que esse grupo de indivíduos também são seres biopsicossociais. Uma criança que completa seu crescimento e desenvolvimento de maneira integral e saudável tende a manter um comportamento saudável na vida adulta, influenciando na qualidade de vida da população de um modo geral.

Desse modo, a criação de uma política nacional de saúde voltada para a população infantil é um passo bastante significativo para a saúde pública brasileira. Além disso, carrega a grande responsabilidade de chamar a atenção e conduzir ações para um público tão 
negligenciado ao longo da história. Ainda há um longo caminho a percorrer para vencer as iniquidades encontradas no nosso país, sobretudo na primeira infância, porém todos esses passos que já foram dados até hoje representam o fortalecimento e a evolução na saúde pública brasileira.

\section{Eixo "Infância" no Observatório de Análise Política em Saúde: projetos em curso}

Nesta seção final do capítulo, apresentaremos, numa descrição panorâmica, o conjunto de pesquisas que compõem o eixo "Infância" ao interior do Observatório de Análise Política em Saúde; algumas ainda em curso, apresentadas como projetos, outras que já forma finalizadas e que trazem uma visão geral dos resultados alcançados. São projetos realizados por pesquisadores, em sua maioria, do Instituto de Saúde Coletiva da Universidade Federal da Bahia (ISC/ UFBA), assim como alunos do Programa de Pós-Graduação, em seus projetos de tese e dissertação.

De modo geral, os trabalhos estão dirigidos a responder as seguintes questões de investigação: quais as razões para o estabelecimento de uma política que responda à magna causa da infância? Como vem se constituindo no Brasil a chamada cruzada da infância? Por que a persistência dos estudos? Qual a situação atual das políticas? Organiza-se desta forma, ao interior do grupo, três perspectivasanalíticas, quais sejam:

1. Acompanhamento das políticas implantadas nos últimos 10 anos por meio da análise de sua situação atual, bem como seu seguimento até 2017;

2. Estudos de caso e estudos avaliativos sobre políticas específicas no âmbito da saúde integral da criança no Brasil e Bahia;

3. Estudos sócio-históricos sobre a gênese da política voltados a indagar sobre processos sociais subjacentes e suas determinações históricas e estruturais. 
Serão descritos a seguir os principais estudos já conduzidos eprojetos de pesquisa em andamento que compõe o eixo Infância:

+ Pesquisa "Saúde na escola: analisando os caminhos da intersetorialidade"

A intersetorialidade tem sido concebida como uma estratégia importante e necessária para a efetividade das políticas públicas. $\mathrm{Na}$ saúde, está fortemente vinculada à discussão sobre os Determinantes Sociais da Saúde e à Promoção da Saúde. (BUSS, 2000; JACKSON et al., 2007) Na educação, é vinculada à ideia de parceria e colaboração. (BRASIL, 2014; OLIVEIRA; SANTANA, 2010) Entretanto, estudos apontam diversos entraves e desafios para a implementação da intersetorialidade. (FERNANDES, 2005, FERREIRA; VIEIRADA-SILVA, 2005, SANTOS, 2011) No intuito de articular as ações de saúde às ações da educação básica, o PSE é concebido como um programa intersetorial com vistas à melhoria da saúde e da educação dos estudantes das creches e das escolas públicas. (BRASIL, 2007; 2013) Tendo em vista que a intersetorialidade é um princípio do PSE, a presente pesquisa buscou a seguinte investigação: como a intersetorialidade tem sido implementada no PSE? O objetivo da pesquisa foi analisar a ação intersetorial entre os setores saúde e educação utilizada no processo de implementação do PSE.

Tratou-se de uma pesquisa exploratória e descritiva, estudo de caso, realizada em um município da Bahia. Para a produção dos dados, realizaram-se entrevistas semiestruturadas, análise documental e observação, e ao final, optou-se pela triangulação dos dados. A pesquisa envolveu gestores das secretarias municipais de educação e de saúde, o grupo de trabalho intersetorial municipal e profissionais de duas equipes de saúde da família e profissionais de três escolas públicas (duas municipais e uma estadual). Para a análise dos dados, houve a construção do modelo lógico do programa e a elaboração de uma matriz de análise, contendo como categoria analítica a intersetorialidade e as dimensões processo político-gerencial e processo de trabalho. 
Os resultados mostraram que conceitualmente a intesertorialidade é pouco compreendida, como também é pouco praticada, havendo mais justaposição de ações. Na implementação do programa, notou-se uma liderança do setor saúde, tanto no que diz respeito ao processo decisório quanto na alocação e mobilização de recursos como nas atividades que são realizadas nas escolas, os gestores da educação e a equipe escolar têm uma atuação coadjuvante. Identificou-se que as atividades são focadas na prevenção e na assistência à saúde dos estudantes. Os principais achados evidenciam que o PSE fortaleceu a relação entre os dois setores, entretanto, a articulação intersetorial no processo político-gerencial e no processo de trabalho mostrou-se frágil e limitada.

- Pesquisa "O abandono do tratamento no contexto dos cuidados de saúde mental para crianças e adolescentes"

Esse projeto corresponde a uma dissertação de mestrado acadêmico realizada no Programa de Pós-Graduação em Saúde Coletiva. O estudo foi dividido em uma revisão de literatura seguida de estudo empírico.

São poucos e divergentes estudos a respeito do conceito de abandono do tratamento e sobre os fatores predisponentes para este em serviços de saúde mental infanto juvenil. Essa pesquisa teve por objetivo apresentar e discutir quais os conceitos e os principais fatores associados ao abandono do tratamento de saúde mental entre crianças, adolescentes e suas famílias, em publicações científicas.

Trata-se de uma revisão sistemática da literatura que consultou as bases de dados Scientific Electronic Library Online (Scielo), Literatura Latino-Americana e do Caribe em Ciências da Saúde (Lilacs), Medical Literature Analysis and Retrieval System Online (Medline), PubMed e National Center for BiotechnologyInformation(NCBI), ultilizando-se artigos publicados desde o primeiro estudo divulgado em 1956, por meio dos seguintes descritores: "pacientes desistentes do tratamento", "abandono", "dropout" e "desistência do paciente", correlacionados à "saúde mental” e "crianças e adolescentes”. A partir 
da aplicação dos critérios de inclusão e exclusão na busca dos artigos, foram encontrados 326 estudos que versavam sobre o abandono do tratamento em saúde mental, sendo que 34 destes versavam sobre o tema na população de crianças e adolescentes. Dos 34 estudos, 11 foram excluídos por não preencherem os critérios de inclusão delimitados, totalizando 23 estudos selecionados para a análise.

Os resultados encontrados evidenciaram o predomínio de trabalhos quantitativos, com significativas divergências conceituais e sobre os resultados produzidos. Alguns fatores foram levantados com maior recorrência entre os estudos como possivelmente associados ao abandono do tratamento entre crianças e adolescentes, tais como a monoparentalidade esobrecarga do cuidado na figura de um único cuidador; uso abusivo de substâncias psicoativas entre os jovens e seus pais; violência intrafamiliar; baixa escolaridade dos pais; baixo capital econômico; discrepâncias entre as expectativas dos familiares e profissionais quanto ao tratamento; atitudes e comportamentos do terapeuta como empatia e disponibilidade subjetiva; pouca clareza 324 sobre os objetivos e métodos de tratamento; barreiras de acesso e acessibilidade; formas de acolhimento; vinculo terapêutico frágil, dentre outros.

Discute-se que conhecer os possíveis preditores para o abandono do tratamento de saúde mental possibilita que os profissionais possam identificar precocemente pacientes pertencentes ao grupo de risco para abandono, oportunizando-lhes trabalhar preventivamente e mais diretamente aspectos que dificultam a permanência desses pacientes e seus familiares na instituição. Apesar da significativa contribuição que esses estudos têm realizado para a produção no conhecimento, ressaltamos a necessidade de realizar novos estudos de cunho qualitativo junto aos familiares, usuários e profissionais do CAPSi, a fim de compreender melhor a realidade desse espaço de cuidado tão diverso dos modelos de tratamento ofertados em outros países.

O estudo empírico tem por objetivo de analisar os elementos relacionados ao abandono do tratamento em um CAPS para crianças e adolescentes, considerando as experiências de adoecimento e 
de cuidado construídas pelos usuários e familiares em sua busca por tratamento de saúde mental. Foram entrevistados familiares e profissionais que participaram diretamente no cuidado das crianças e adolescentes, no período de sua permanência no serviço, bem como realizado um grupo focal com profissionais do CAPSi. Como resultados do estudo, identifica-se dificuldades dos familiares quanto à compreensão das novas propostas de cuidados em saúde mental propostas pelo CAPS, as quais podem interferir diretamente na continuidade do cuidado do familiar no serviço. Identificou-se que os cuidados das crianças e adolescentes em sofrimento psíquico estão centrados na figura de um único cuidador do sexo feminino. Duas das três famílias demonstraram dificuldade em administrar o papel de cuidador e conciliá-lo às exigências das condições concretas de vida. A ênfase no hospital psiquiátrico enquanto espaço de eleição de cuidados ainda pode ser observada no discurso das famílias.

Como aspectos relacionados à adesão e ao abandono do tratamento no serviço, identificam-se: aspectos relacionados às características familiares (escolaridade dos pais, monoparentalidade, escassa rede de suporte afetiva, discrepâncias entre as expectativas dos familiares e profissionais quanto ao tratamento, baixo capital econômico, incompreensão sobre o modelo de tratamento, baixa crença na efetividade do tratamento, sentimento de desesperança, valorização do tratamento farmacológico etc); aspectos relacionados ao paciente e ao processo de sofrimento (agressividade, gravidade do caso, uso de spas, piora do quadro etc); aspectos ligados às características dos serviços de saúde (fragilidade do vínculo terapêutico, ausência de grupos de apoio para os pais, distância entre o local de moradia e o serviço, fontes de encaminhamento, pouca clareza sobre os objetivos e métodos de tratamento); e aspectos comunitários tais como violência urbana, foram alguns dos motivos apontados para o abandono. Discute-se que o abandono do tratamento, nos três casos, relacionou-se a diversos fatores, nem sempre evidentes para os familiares. 
- Pesquisa "Análise da Implantação da Rede Cegonha: desafios e perspectivas para redução da sífilis congênita na atenção básica"

O estudo, resultado de projeto de dissertação de Mestrado Acadêmico em Saúde Coletiva do Programa de Pós-Graduação em Saúde Coletiva da UFBA, teve por objetivo estimar o grau de implantação das ações dirigidas ao controle da sífilis congênita no prénatal, no âmbito da Rede Cegonha, na atenção básica no município de Palmas. Para realização da pesquisa, foi utilizada uma abordagem avaliativa do tipo análise de implantação do componente pré-natal da Rede Cegonha no município de Palmas, em Tocantins. Tendo em vista explicitar a intervenção, foi elaborado um modelo lógico do programa por meio de consultas a documentos oficiais, portarias que regulamentam o programa, assim como realização de entrevistas com membros da equipe da ESF das unidades básicas de saúde envolvidos com o processo de implantação da Rede Cegonha em Palmas. Para classificação dos achados, utilizou-se uma matriz de implantação derivada do modelo lógico, aplicada em oito unidades básicas de saúde selecionadas por sorteio.

Os resultados desse estudo demonstraram que o grau de implantação das ações dirigidas ao controle da sífilis congênita, no âmbito da Rede Cegonha, na atenção básica no município de Palmas, encontra-se parcialmente implantado. Revelou-se ainda a baixa adesão dos parceiros das gestantes, tanto para realização do teste rápido quanto para o tratamento da sífilis, o que contribui para o aumento da transmissão vertical da sífilis. Recomenda-se ações que favoreçam a inserção do parceiro no pré-natal e uma maior integração entre as atividades desenvolvidas na implantação da saúde da mulher e saúde do homem. 
- Projeto "Espaço social de luta pela infância: gênese e transformações"

O estudo sócio-histórico interessa-se pelas seguintes questões: por que e como o Estado brasileiro assume o problema da mortalidade infantil e a atenção à infância como sua responsabilidade? Qual a situação da implantação das políticas de atenção à infância ao longo do período 2003-2013? Possui como objetivos: analisar a emergência e transformações das políticas de saúde materno infantil no Brasil, identificando suas condições de possibilidade históricas; analisar a gênese e evolução de espaços profissionais especializados nos problemas da infância no Brasil.

O estudo será apoiado no referencial teórico da sociologia reflexiva de Bourdieu (1996), autores que discutem a sociogênese das políticas sociais (PINELL, 2010), bem como naqueles que contribuem para a análise da infância numa perspectiva sócio-histórica. (ARIÈS, 1986) A abordagem sócio-histórica interessa-se em analisar os agentes e instituições envolvidas na emergência da política bem como as condições históricas de possibilidade que resultaram em sua constituição.

Trata-se de estudo histórico sobre a gênese e transformações das políticas de saúde materno infantil no Brasil, no período compreendido entre o início dos anos 1970, período em que há a formulação de políticas e programas voltados à infância, como Programa Nacional de Imunizações (1973), Programa nacional de saúde materno-infantil (1975), Programa Nacional de Aleitamento Materno (1981). Será realizado igualmente um acompanhamento das políticas implantadas nos últimos 10 anos (2003-2013), a partir de diversos estudos de caso sobre políticas específicas no âmbito da saúde integral da criança no Brasil e Bahia. Durante a fase exploratória do estudo, esse período da análise será melhor delimitado por meio da análise documental sobre as ações do Estado brasileiro relativas à saúde da infância em períodos anteriores. 
Para produção dos dados, em um primeiro momento, será realizado um estudo exploratório buscando identificar os principais marcos históricos e agentes relacionados com o objeto da pesquisa, bem como os informantes chave. Para investigar a trajetória dos agentes, serão realizadas entrevistas em profundidade (presenciais ou por Skype) com os informantes chave, responsáveis pela implantação das políticas. Para os já falecidos, serão consultadas biografias publicadas, entrevistas outrora concedidas ou seus descendentes. Será realizada ainda consulta aos currículos dos diversos agentes, em particular aqueles disponíveis na Plataforma Lattes. ${ }^{1}$ Para análise das condições de possibilidade de surgimento das políticas, serão consideradas as fontes bibliográficas e documentais que permitam reconstituir as principais conjunturas política, econômica e social da época. Serão realizadas ainda buscas sistemáticas na internet e nos acervos dos jornais e revistas especializadas, tais como a Gazeta Médica da Bahia, ${ }^{2}$ o Estado de São Paulo, ${ }^{3}$ a Folha de S. Paulo ${ }^{4}$ e outros que as fase exploratória da investigação indicar.

- Projeto "Análise da situação atual da Política Nacional de Atenção Integral da Criança”

A política nacional de saúde voltada para a população infantil é um passo bastante significativo para a saúde pública brasileira. No entanto, ainda há um longo caminho a percorrer para vencer as iniquidades encontradas no nosso país, sobretudo na primeira infância. Uma forma importante de acompanhar e mensurar os avanços na promoção de saúde na infância através de uma política de saúde pública é acompanhar todas as notícias e portarias disponíveis sobre o assunto. Portanto, destaca-se a importância da monitorização de no-

\footnotetext{
1 http://lattes.cnpq.br/.

2 http://www.gmbahia.ufba.br/index.php/gmbahia/login.

3 http://acervo.estadao.com.br/

4 http://acervo.folha.com.br/
} 
tícias sobre a Política Nacional de Atenção Integral da Criança para registrar os seus avanços com o decorrer dos anos.

O Projeto de Monitorização das notícias sobre a Política Nacional de Atenção Integral da Criança tem como principais objetivos registrar informações coletadas em matriz de análise para sistematização de documentos e notícias bem como realizar uma descrição das políticas identificadas, destacando agentes envolvidos, objetivos, estratégias; cobertura e implantação; principais resultados alcançados; controle social, bem como outras categorias de análise propostas. A estratégia metodológica da pesquisa inclui o levantamento e sistematização de documentos sobre políticas de saúde voltadas ao público materno infantil nos sites de referência do MS, das Secretarias Estaduais de Saúde, das Secretarias Municipais de Saúde (SMS), do Conselho Nacional de Secretários de Saúde (Conass), do Conselho Federal de Medicina (CFM), do Centro Brasileiro de Estudos de Saúde (Cebes), da Associação Brasileira de Saúde Coletiva (Abrasco), entre outros.A pesquisa encontra-se em fase de registro das informações lançadas após o decreto da portaria assinada pelo Ministro Arthur Chioro da Política Nacional de Assistência a Saúde Integral da Criança.

- Projeto "A violência doméstica contra crianças como problema social e de saúde: experiências familiares e respostas sociais em um bairro popular de Salvador/BA"

Estudos dos últimos anos sobre violência contra crianças têm abordado, por um lado, as consequências do agravo e os fatores de proteção e de risco presentes no seu ambiente (ALVES; EMMEL, 2008; DE ANTONI; KOLLER, 2000, 2010; GABATZ et al., 2010a; 2010b; MARTINS et al., 2007) e, por outro, a avaliação dos serviços de atenção e as perspectivas dos diferentes profissionais sobre sua atuação diante desse problema. (BAZON; FALEIROS, 2013; GOMES et al., 2002; SILVA, L.; FERRIANI; SILVA, M., 2012) Destaca-se a relevância da ação interinstitucional e intersetorial como facilitador de uma intervenção efetiva. As questões de como 
a família experiencia o fato, qual a sua trajetória entre os diferentes serviços e quais as situações promotoras de vulnerabilidade ou resiliência nesse processo permanecem sem uma resposta que abarque a complexidade do fenômeno.

Nesse sentido, esse projeto tem como objetivo geral analisar problemas e necessidades sociais e de saúde de famílias com história de violência doméstica contra crianças, destacando serviços de proteção e respostas sociais empreendidas em um bairro popular de Salvador/BA. Especificamente, pretende descrever a trajetória de famílias no cuidado a crianças com história de violência doméstica; caracterizar os serviços de proteção social e da saúde utilizados no cuidado a crianças em situação de violência doméstica; identificar situações de vulnerabilidade, resiliência, risco e proteção em crianças submetidas à violência doméstica; e analisar possíveis linhas de cuidado e/ou itinerários terapêuticos percorridos por crianças vítimas de violência doméstica.

Trata-se de uma pesquisa exploratória, qualitativa, com dese330 nho de estudo de caso. (YIN, 2001) Considerando a complexidade do fenômeno violência, foi feita uma escolha pela triangulação metodológica, combinando entrevistas semiestruturadas com 5 famílias e 10 representantes de instituições da rede de proteção referidas pelas famílias nas entrevistas. Os resultados serão analisados à luz da bioecologia do desenvolvimento humano (BRONFENBRENNER, 1996; KOLLER; DE ANTONI, 2004), especificamente seus conceitos de contextos de desenvolvimento, fatores de risco e fatores de proteção. Adicionalmente ao modelo, serão utilizados os conceitos adjacentes de resiliência e vulnerabilidade (SELIGMAN; CSIKSZENTMIHALYI, 2000; YUNES; MIRANDA; CUELLO, 2004) e de linha de cuidado (MALTA; MERHY, 2010) e itinerários terapêuticos. (ALVES; SOUZA, 1999) 
- Projeto "Espaço de luta e de atenção à criança com deficiência na Bahia"

Esse projeto parte da seguinte pergunta de investigação: como está o movimento político em defesa dos direitos das crianças com deficiência na Bahia? Como está organizada a rede de atenção à criança portadora de deficiência na Bahia? Tem como objetivo registrar a situação atual do movimento político em defesa dos direitos das crianças com deficiência na Bahia e a sua rede de atenção e de cuidado a essas crianças. De forma específica visa identificar os atores sociais envolvidos na luta pelos direitos das crianças com deficiência na Bahia; identificar as políticas públicas que contribuíram para a garantia dos direitos das crianças portadoras de deficiência e mapear a rede de atenção e de cuidado à criança com deficiência na Bahia.

Como estratégia metodológica serão realizadas entrevistas em profundidade com os agentes protagonistas na luta em defesa das crianças com deficiência na Bahia, considerando os envolvidos no movimento, na assistência a essas crianças, além de visitar os serviços de cuidado e de realizar pesquisas em documentos, livros e documentários.

\section{Referências}

ALVES, P. C.; SOUZA, I. M. Escolha e avaliação de tratamento para problemas de saúde: considerações sobre o itinerário terapêutico. In: RABELO, M. C.; ALVES, P. C.; SOUZA, I. M. Experiência de doença e narrativa. Rio de Janeiro: Ed. Fiocruz; 1999. p. 125-138.

ANDRADE, L. B. P. Educação infantil: discurso, legislação e práticas institucionais. São Paulo: Ed. UNESP: Cultura Acadêmica, 2010.

AQUINO, R.; OLIVEIRA, N. F. de; BARRETO. M. L. Impact of the family healthprogram on infant mortality in Brazilian municipalities. American Journal of Public Health, New York, p. 87-93, 2009. 
ARAÚJO, J. P. et al. História da saúde da criança: conquistas, políticas e perspectivas. Revista Brasileira de Enfermagem, Brasília, DF, v. 67, n. 6, p. 1000-1007, nov./dez. 2014.

ARIÈS, P. História social da criança e da família. Tradução de Dora Flaksman. 2.ed. Rio de Janeiro: Guanabara, 1986.

BARBOSA, M. C. S. Por amor e por força: rotinas na educação infantil. Porto Alegre: Artmed, 2006.

BAZON, M. R.; FALEIROS, J. M. Identificação e notificação dos maustratos Infantis no Setor Educacional. Paidéia, Ribeirão Preto, v. 23, n. 54, abr. 2013.

BRASIL. Decreto no 6.286, 5 de dezembro de 2007. Institui o Programa Saúde na Escola - PSE, e dá outras providências. Diário Oficial [da] República Federativa. Brasília, DF, 6 dez. 2007. Disponível em<http:// www.planalto.gov.br/ccivil_03/_ato2007-2010/2007/decreto/d6286. htm>. Acesso em: 10 ago. 2015.

BRASIL. Lei n. 8.069, de 13 de julho de 1990. Dispõe sobre o Estatuto da Criança e do Adolescente e dá outras providências. Diário Oficial [da] República Federativa doBrasil. Brasília, DF, 16 jul.1990. p. 13.563.

BRASIL. Ministério da Saúde. Programa nacional de incentivo ao aleitamento materno - PNIAM. Brasília, DF,1991.

BRASIL. Ministério da Saúde. Instrumento gerencial da saúde da mulher, da criança e do adolescente: avaliação, sistema de informação e programação. Brasília, DF, 1995.

BRASIL. Portaria no 399, de 22 de fevereiro de 2006. Divulga o Pacto pela Saúde 2006 - Consolidação do SUS e aprova as Diretrizes Operacionais do Referido Pacto. Diário Oficial [da] União, Brasília, DF, 23 fev. 2006.

BRASIL. Ministério da Saúde. Secretária de Politicas ed Saúde. Saúde da criança: acompanhamento do crescimento e desenvolvimento infantil. A. Normas e Manuais Técnicos. Brasília, DF, 2002a. (Série Cadernos de Atenção Básica, n. 110). 
BRASIL. Ministério da Saúde. Secretaria de Atenção à Saúde.

Departamento de ações programáticas estratégicas. Pacto Nacional pela redução da morte materna e neonatal. Brasília, DF, 2004a.

BRASIL. Ministério da Saúde. Secretaria de Atenção à Saúde.

Departamento de Ações Programáticas Estratégicas. Área técnica da saúde da criança e aleitamento materno: agenda de compromissos para a saúde integral da criança e redução da mortalidade infantil. Brasília, DF, 2004b.

BRASIL. Ministério da Saúde. Mais saúde direito de todos, 2008-2011. 2. ed. Brasília, DF, 2008.

Brasil. Ministério do Desenvolvimento Social. Brasil Carinhoso. Brasília, DF, 2015a. Disponível em: <http://mds.gov.br/brasil-sem-miseria/ acesso-a-servicos/brasil-carinhoso >. Acesso em: 24 jul. 2016.

BRASIL. Ministério da Educação. Plano Nacional da Educação - PNE 2014-2024. Brasília, DF, 2014. Disponível em: <http://pne.mec.gov.br>. Acesso em: 10 set. 2015.

BRASIL. Ministério da Saúde. Portaria Interministerial no 1.413, de 10 de julho de 2013. Redefine as regras e critérios para adesão ao Programa Saúde na Escola (PSE) por Estados, Distrito Federal e Municípios e dispõe sobre o respectivo incentivo financeiro para custeio de ações. Diário Oficial da União. Brasília, DF, 2013. Disponível em: <http:// bvsms.saude.gov.br/bvs/saudelegis/gm/2013/pri1413_10_07_2013. htm>. Acesso em: 20 ago. 2015.

BRASIL.Ministério da Saúde Portariano 1.130, de 5 de agosto de 2015. Institui a Política Nacional de Atenção Integral à Saúde da Criança (PNAISC) no âmbito do Sistema Único de Saúde (SUS). Diário Oficial da União. Brasília, DF, 5 out. 2015b.

BRASIL. Ministério da Saúde. Secretaria de Políticas de Saúde. Área da Saúde da Criança. Programas e Projetos da Saúde da Criança: responsabilidades compartilhadas em benefício das crianças brasileiras. Revista Brasileira de Saúde Materno Infantil, Recife, v. 2, n. 2, p. 193-196, maio/ago. 2002b.

BOURDIEU, P. As regras da arte: gênese e estrutura do campo literário. Lisboa: Presença, 1996. 
BUSS, P. M. Promoção da saúde e qualidade de vida. Revista Ciência e Saúde Coletiva, Rio de Janeiro, v. 5, n. 1, p. 163-177, 2000.

BRONFENBRENNER, U. A ecologia do desenvolvimento humano: experimentos naturais e planejados. Porto Alegre: Artes Médicas, 1996.

CABRAL, I. E.; AGUIAR, R. C. B. de. As políticas públicas de atenção à saúde da criança menor de cinco anos: um estudo bibliográfico. Revista Enfermagem UERJ, Rio de Janeiro, v. 11, p. 285-291, 2003.

COUTO, M. C. V.; DUARTE, C. S.; DELGADO, P. G. G. A saúde mental infantil na Saúde Pública brasileira: situação atual e desafios. Revista brasileira de psiquiatria, São Paulo, v. 30, n. 4, p. 384-389, dez. 2008.

CUNHA, C.L.F.et al . O uso de serviços de atenção primária à saúde pela população infantil em um estado do nordeste brasileiro. Caderno de Saúde Coletiva, Rio de Janeiro, v. 21, n. 2, p. 115-120, June 2013.

CUNHA, A. C. B. da; BENEVIDES, J. Prática do psicólogo em intervenção precoce na saúde materno-infantil. Psicologia em Estudo, Maringá, v. 17, n. 1, p. 111-119, mar. 2012.

334 DE ANTONI, C.; KOLLER, S. H. A visão de família entre as adolescentes quesofreramviolência intrafamiliar. Estudos de Psicologia, Natal, v. 5, n. 2, p. 2000.

DE ANTONI, C.; KOLLER, S. H. Uma família fisicamente violenta: uma visão pela teoria bioecológica do desenvolvimento humano. Temas em Psicologia, Ribeirão Preto,v. 18, n. 1, p. 2010.

FERNANDES, D. M. Análise da articulação intersetorial na implementação de um programa social em nivel local: experiência do município de Petrópolis/ RJ. 2005. Dissertação (Mestrado em Saúde Pública) Fundação Oswaldo Cruz, Escola Nacional de Saúde Pública, 2005.

FERREIRA, V.S.C, VIEIRA-DA-SILVA, L. M. Intersetorialidade em saúde: um estudo de caso. In: HARTZ, Z. M. (Org.). Avaliação em saúde: dos modelos teóricos à prática na avaliação de programas e sistemas de saúde. EDUFBA, 2005. p. 103-150. 
FINKLER, A. L. et al. $\mathrm{O}$ acesso e a dificuldade na resolutividade do cuidado da criança na atenção primária à saúde. Acta Paulista de Enfermagem. São Paulo, v. 27, n. 6, p. 548-53, 2014.

FIGUEIREDO, G. L. A.; MELLO, D. F. de. Atenção à saúde da criança no Brasil: aspectos da vulnerabilidade programática e dos direitos humanos. Revista Latino-Americana de Enfermagem, Ribeirão Preto, v. 15, n. 6, p. dec. 2007.

GABATZ, R. I. B. et al. Fatores relacionados à institucionalização: perspectivas de crianças vítimas de violência intrafamiliar. Revista Gaúcha de Enfermagem, Porto Alegre, v. 31, n. 4, p. 670-677, dez. 2010a.

GABATZ, R. I. B.et al. O significado de cuidado para crianças vítimas de violência intrafamiliar. Escola Anna Nery, Rio de Janeiro,v.14, n.1, p. 135-142, mar. 2010b.

GOMES, R., et al. A abordagem dos maus-tratos contra a criança e o adolescente em uma unidade pública de saúde. Ciência e Saúde Coletiva, São Paulo, v. 7, n. 2, p. 275-283, 2002.

JACKSON, F.S. et al. Integrated health promotion strategies: a contribution to tackling current and future health challenges. Health Promotion International, Oxford, v. 21, n. 1, p. 75-83, 2007

JAVEAU, C. Criança, infância(s), crianças: que objetivo dar a umaciência social da infância? Educação e Sociedade, Campinas, v. 26, n. 91, p. 379-89, ago. 2005.

KOLLER, S. H.; DE ANTONI, C. Violência intrafamiliar: uma visão ecológica. In: KOLLER, S. H. (Org.). Ecologia do desenvolvimento humano: pesquisa e intervenção no Brasil. São Paulo: Casa do Psicólogo, 2004. p. 293-310.

LERNER, R. et al . A Psicologia na articulação entre os âmbitos coletivo e psíquico: construção de uma política pública em saúde de cuidado com o desenvolvimento. Psicologia: Ciência e Profissão, Brasília, DF, v. 33, p. 100-111, 2013. Edição especial.

MALTA, D.C.; MERHY, E.E.The path of the line of care from the perspective of nontransmissiblechronic diseases. Interface. Botucatu, v.14, n. 34, p. 593-605, jul./set. 2010. 
MARQUES, E.P. Infância e políticas públicas: a intersetorialidade como estratégia de atenção integral à saúde da criança. Sociedade em Debate, Pelotas, v. 16, n. 1, p. 107-120, jan./jun.2010.

MARTINS, C. S. et al. Family dynamics from the perspective of parents and children involved in domestic violence against children and adolescents. Revista Latino-Americana de Enfermagem, Ribeirão Preto, v. 15 , n. 5, p. 889-894, Oct. 2007

NOVAES, M. H. D.A puericultura em questão. 1979. Dissertação (Mestrado em Medicina) - Faculdade de Medicina, Universidade de São Paulo, São Paulo, 1979.

OLIVA, J. C. G. A.; KAUCHAKJE, S. As políticas sociais públicas e os novos sujeitos de direitos: crianças e adolescentes. Revista katálysis, Florianópolis, v. 12, n. 1, p. 22-31, Jun. 2009.

OLIVEIRA, R. P.; SANTANA, W. (Org.). Educação e federalismo no Brasil: combater as desigualdades, garantir a diversidade. Brasília, DF: Unesco, 2010.

PIMENTA, T. A. M.; ROCHA, R.; MARCONDES, N. A.V. Políticas Públicas de intervenção na obesidade infantil no Brasil: uma breve análise da política nacional de alimentação e nutrição e política nacional de promoção da saúde. Unopar científica: Ciências biológicas e da saúde, Londrina, v. 17, n. 2, p. 139-146, 2015.

PINELL, P. Análise sociológica das políticas de saúde. Rio de Janeiro: Ed. Fiocruz, 2010.

UNICEF.A Convenção sobreos Direitos da Criança: adoptada pela Assembleia Geral nas Nações Unidasem 20 de Novembro de 1989 e ratificada por Portugalem 21 de Setembro de 1990.[2004?]. Disponível em: <http://www.unicef.pt/docs/pdf_publicacoes/convencao_ direitos_crianca2004.pdf >. Acesso em: 15/08/2014.

QVORTRUP, J. A infância enquanto categoria estrutural. Educação e Pesquisa, São Paulo, v. 36, n. 2, p. 631-644, ago. 2010

RAMOS, B. de C.; MAIA, L. C. Cárie tipo mamadeira e a importância da promoção de saúde bucal em crianças de 0 a 4 anos. Revista de 
Odontologia da Universidade de São Paulo, São Paulo, v. 13, n. 3, p. 303-311, 1999.

RASELLA D. et al.Effect of a conditional cash transfer programme on childhood mortality: a nationwide analysis of Brazilian municipalities. The Lancet, London, v. 382, n. 9886, p. 57-64, July 2013.

ROCHA, S. M. M. et al. Estudo da assistência integral à criança e ao adolescente através da pesquisa qualitativa. Revista Latino-Americana de Enfermagem, Ribeirão Preto, v. 6, n. 5, p. 5-15,1998.

SANTOS, N. N. A intersetorialidade como modelo de gestão das políticas de combate à pobreza no Brasil: o caso do programa bolsa família no município de Guarulhos. 2011. Dissertação (Mestrado em Administração) - Escola de Administração, Fundação Getúlio Vargas, São Paulo, 2011.

SARMENTO, M. J. Geração e alteridade: interrogação a partir da Sociologia da Infância.Educaçãoł Sociedade, Campinas, v. 26, n. 91, p. 361-78, maio/ago. 2005.

SELIGMAN, M. E. P.; CSIKSZENTMIHALYI, N. Apositive psychology: an introduction. American Psychologist, Washington, v. 55, n. 1, p. 5-14, 2000

SILVA, L. M. P. da; FERRINI, M. das G. C.; SILVA, M. A. I. O Poder Judiciário como última instância de proteção às crianças e aos adolescentes: ações intersetoriais, investimento em recursos humanos e estruturação dos serviços. Revista Latino-Americana de Enfermagem," Ribeirão Preto, v. 20, n. 3, jun. 2012.

SOUZA, M. C; OLIVEIRA, I.C. Assistência de enfermagem à criança hospitalizada: breve retrospectiva. Revista Brasileira de Enfermagem, Brasília, DF, v. 57, n. 2, p. 247-249, mar./abr. 2004.

TYRREL,M. A. R. B. Programas nacionais de saúde materno infantil: impacto social e inserção da enfermagem. Revista Brasileira de Enfermagem,Brasília, DF, v. 1, n. 1, p. 119-134, 1997.

UNICEF. Declaração Universal Dos Direitos Das Crianças. 1959.

Disponível em: <http://bvsms.saude.gov.br/bvs/publicacoes/ declaracao_universal_direitos_crianca.pdf>. Acesso em: 15 ago. 2014. 
VAILATI, L. L. A morte menina. Infância e morte infantil no Brasil dos oitocentos (Rio de Janeiro e São Paulo). São Paulo: Alameda, 2010, VICTORA, C. G. Intervenções para reduzir a mortalidade infantil préescolar e materna no Brasil. Revista Brasileira de Epidemiologia, São Paulo, v. 4, n. 1, p. 3-53, 2001.

VICTORA, C. G. et al. Saúde das mães e crianças no Brasil: progressos e desafios. The Lancet, London, p. 32-46, maio 2011.

WARDE, M. J. Repensando os estudos sociais de história da infância no Brasil. Perspectiva, Florianópolis, v. 25, n. 1, p. 21-39, jan. 2007.

YIN, R. K. Estudo de caso: planejamento e métodos. Porto Alegre:

Bookman, 2001.

YUNES, M. A. A.; MIRANDA, A. T.; CUELLO, S. E. S. Um olhar ecológico para os riscos e as oportunidades. In: KOLLER, S. H. (Ed.). Ecologia do desenvolvimento humano: pesquisa e intervenção no Brasil. São Paulo: Casa do Psicólogo, 2004. p.197-218.

ZANOLLI, M. L.; MERHY, E. E. A pediatria social e as suas apostas reformistas. Caderno de Saúde Pública, Rio de Janeiro, v. 17, n. 4, p. 977-987, ago. 2001. 